Usages du français et pratiques d'enseignement en Europe balkanique, centrale et orientale - Grèce,

Serbie, Bulgarie, Moldavie, Hongrie, Allemagne, Russie - XVIIle - XXe siècles

\title{
Le français en Moldavie. Entre héritage, tradition et mondialisation
}

Le français en Moldavie. Entre héritage, tradition et mondialisation

\section{Olga Turcan}

\section{OpenEdition}

Journals

\section{Édition électronique}

URL : https://journals.openedition.org/dhfles/4245

DOI : $10.4000 /$ dhfles.4245

ISSN : 2221-4038

\section{Éditeur}

Société Internationale pour l'Histoire du Français Langue Étrangère ou Seconde

Édition imprimée

Date de publication : 1 juin 2015

Pagination : 195-208

ISSN : 0992-7654

\section{Référence électronique}

Olga Turcan, «Le français en Moldavie. Entre héritage, tradition et mondialisation », Documents pour I'histoire du français langue étrangère ou seconde [En ligne], 54 | 2015, mis en ligne le 13 décembre 2017, consulté le 25 mars 2023. URL : http://journals.openedition.org/dhfles/4245 ; DOI : https://doi.org/ $10.4000 /$ dhfles. 4245

Ce document a été généré automatiquement le 25 mars 2023

Tous droits réservés 


\title{
Le français en Moldavie. Entre héritage, tradition et mondialisation
}

Le français en Moldavie. Entre héritage, tradition et mondialisation

\author{
Olga Turcan
}

1 Thèse de doctorat en sciences du langage sous la direction de Dominique Huck (Université de Strasbourg) et d'Ana Gutu (Université Libre Internationale de Moldavie), soutenue le 27 mars 2014 à l'Université de Strasbourg. 2 vol. ( $375+202$ pages).

2 Ce travail de thèse interroge la place du français en Moldavie ${ }^{1}$ avant et après l'indépendance du pays en 1991, dans des contextes d'héritage historique, de tradition d'enseignement et de mondialisation. Il questionne également l'existence d'une politique linguistique à l'égard du français. Les acteurs de la francophonie, qu'ils soient endogènes ou exogènes, et leur action à l'égard du français font partie du champ de recherche. En clair, ce travail cherche à faire état des lieux du français en contexte moldave.

\section{1. État des recherches sur le sujet}

L'objet de cette recherche se situe à la croisée de plusieurs champs disciplinaires (histoire, sociolinguistique, politique linguistique).

4 Les publications annuelles du colloque international "Francopolyphonie », organisé par l'Institut de recherches philologiques et interculturelles de Chisinau, fournissent depuis 2006 une somme de contributions sur la langue française et la francophonie en Moldavie. Dans des disciplines différentes, Ion Gutu, Ana Gutu, Pierre Morel et bien d'autres chercheurs questionnent en permanence la langue française. Pourtant, il n'existe aucune étude qui traite de manière plus ample les problématiques évoquées plus haut.

5 Du côté des recherches françaises ou francophones, les auteurs évoquent très peu, par une phrase ou une énumération rapide des pays de l'Europe centrale et orientale, la situation du français en Moldavie. Truchot fait également observer que « la situation du 
français à l'époque contemporaine dans ces pays [la Moldavie, la Roumanie, ...] est encore peu étudiée » $(2000: 73)$.

6 Ainsi, il paraissait étonnant de constater que l'on n'accordait pas beaucoup de place dans les recherches sur la francophonie, à un pays qui semblait garder le français en première position dans l'enseignement des langues étrangères, situation unique en Europe, depuis une quinzaine d'années au moins. On peut penser que cette absence soit due au fait qu'il s'agit d'un État encore jeune et méconnu qui, de par son histoire complexe, a traversé de nombreuses transformations politiques (partie de la principauté de Moldavie, goubernie russe, partie de la Roumanie, république soviétique) jusqu'à son indépendance en 1991.

7 Le fait de faire partie de ceux qui commencent à s'intéresser à la Moldavie par cet angle comporte quelques difficultés dans la mesure où il y a fort peu de documents sur le français en Moldavie, particulièrement pour la période du XVIII ${ }^{e}$ au début du XXe siècle. Des éclairages sur ce sujet ont pu être apportés grâce aux travaux de Ciobanu, Ghervas, Iorga, Lemny, Camariano-Cioran entre autres chercheurs. Pour le cadre plus général sur l'histoire de la diffusion du français en Europe, nous nous appuyons sur les ouvrages classiques de Brunot puis ceux de Coste ainsi que de Rey, Duval, Siouffi (ouvrage collectif) qui ont constitué un appui important. Les recherches de Gutu I., Morel, Gutu A., Parmentier sur la francophonie moldave après 1991 ont fourni un regard pluriel (historique, politique, culturel) sur un objet en évolution.

\section{Méthodologie}

8 Ces premiers constats sur l'état de la recherche ont mené vers un travail de recherche et d'analyse documentaire (produits discursifs issus de l'archive historique et/ou proférés par des acteurs politiques), ainsi que vers un travail d'enquête (entretiens et questionnaires avec des acteurs, usagers ou politiques). Recueillir des données sur le français en Moldavie a déterminé la mise en place de plusieurs démarches, auprès des personnes susceptibles d'offrir des éléments de réflexion, notamment, des Moldaves, acteurs privés ou représentants des institutions éducatives et de la francophonie. En 2009, une enquête sur la langue française a été réalisée sur la base de deux questionnaires destinés à deux types de public: le «tout public », c'est-à-dire tout citoyen moldave locuteur ou non de français, et le public de l'Alliance française de Moldavie et de son réseau, bénéficiaire des activités que l'association organise.

Comme le champ éducatif présentait un intérêt central pour le questionnement sur la place du français, une recherche documentaire a été effectuée en 2010 en Moldavie, dans les archives du ministère de l'Éducation. De nombreux documents de la période 1975-2010 ont été examinés et retenus pour l'analyse: manuels de français, programmes scolaires, documents relatifs à la formation des professeurs de français, aux bourses d'études pour le perfectionnement du français, etc. Comme le positionnement des langues étrangères, particulièrement de l'anglais et du français, allait vers une inversion en faveur de l'anglais (ou une rupture pour le français?) en 2011, les deux années suivantes, 2012 et 2013, ont été également traitées.

En 2012, des entretiens ont été effectués avec la responsable des langues étrangères du ministère de l'Éducation, ainsi qu'avec des acteurs du ministère sur le terrain, dans les écoles (directeur d'école, professeur de français), ainsi que des échanges avec deux représentants de l'antenne de l'AUF à Chisinau. Après le travail important sur des 
documents élaborés par le ministère de l'Éducation, certains aspects liés à l'enseignement du français, ainsi qu'aux projets des acteurs de la francophonie, ont pu être clarifiés à travers des entretiens avec des interlocuteurs du ministère de l'Éducation, ainsi qu'avec des anciens élèves des classes bilingues.

11 Si notre travail de recherche pour analyser la situation du français en Moldavie aujourd'hui a pris la forme d'entretiens et d'enquête documentaire, une approche historique s'est révélée nécessaire pour comprendre cette situation, héritée de longs siècles de pratiques de la langue française. C'est cette approche - qui fait l'objet de la première partie de la thèse - que nous allons présenter ici, explorant les différentes voies de diffusion de cette langue et ses statuts successifs; nous mentionnerons très brièvement comment dans les deuxième et troisième parties de la thèse nous avons interrogé l'existence de politique(s) linguistique(s) à l'égard du français à travers une lecture contextualisée de textes officiels, de propos d'acteurs éducatifs et sociaux, et l'action des acteurs de la francophonie en faveur du français.

\section{Voies et acteurs de la diffusion du français en Moldavie}

12 La «présence » du français en Moldavie nécessitait une contextualisation, d'abord historique et diachronique, démarche qui fait remonter au XVIII siècle pour retracer sa situation jusqu'à aujourd'hui. C'est dans ce sens qu'ont été étudiées les voies de diffusion du français en Moldavie et les acteurs de sa médiation, tenant compte de sa position géographique, ainsi que de son histoire complexe. L'étude de nombreuses sources historiques permet de voir que les changements politiques traversées par la Moldavie influencent la situation du français, son statut et ses fonctions. Au XVIII ${ }^{e}$ siècle, tant en Moldavie, partie de la Principauté de la Moldavie que dans toute l'Europe, c'est la classe dominante qui adopte le français. Les princes phanariotes et les familles de nobles roumains se focalisent autour du français considéré non seulement comme "symbole de la culture " et outil de communication, mais aussi et surtout comme un instrument de pouvoir, un signe de distinction et une marque de prestige. Son statut symbolique de langue universelle s'étend et se réduit en même temps à une élite, privilégiée et riche.

13 De nombreux acteurs ont contribué à la diffusion du français sur ce territoire : les Phanariotes, les Français - de passage ou émigrés -, les enseignants de français de différentes origines, les Roumains eux-mêmes, les Russes francisés. Nombreux sont les secteurs qui adoptent et font circuler cette langue : l'éducation, le théâtre, la presse, le commerce, l'armée, la diplomatie, la littérature et la liste pourrait être allongée.

\subsection{Médiation par les Phanariotes}

14 Plusieurs historiens roumains affirment que l'apport des Phanariotes ${ }^{2}$ à la diffusion de la langue et de la culture françaises en Moldavie et Valachie a été capital. Dans son article intitulé "Sur la pénétration des idées de l'Occident dans le Sud-Est de l'Europe aux XVII et XVIII ${ }^{e}$ siècles ", publié en 1924 à Paris, Nicolae Iorga ${ }^{3}$ attribue le mérite de la diffusion de la langue et de la culture française en Moldavie et Valachie à quelques Phanariotes comme Constantin Mavrocordato, Alexandre Ypsilanti (Hypsilantès) et Constantin Mourousi. 
L'année 1776 est celle où la première mention de la langue française est faite dans un acte officiel du domaine de l'enseignement, émis par le prince de Valachie, Alexandre Ypsilanti ${ }^{4}$. Dans les faits, l'enseignement du français et en français (les mathématiques, la philosophie) se faisaient déjà bien avant, à l'Académie princière de Bucarest. Une évolution similaire a lieu dans l'enseignement du français en Moldavie lors du règne de Constantin Morousi (1777-1782) qui, à l'instar de son homologue de Valachie, est préoccupé par la réorganisation de l'Académie princière de Iassi. Les Académies constituaient un cadre institutionnel de diffusion de la langue française, un cadre sélectif qui élargissait l'accès aux études à d'autres couches sociales que les nobles (citadins, marchands, artisans, etc.) et même à d'autres peuples que celui des Principautés roumaines dans la région balkanique. À cette époque-là, les études dans les académies princières représentaient un gage de qualité et par conséquent de réussite ultérieure dans la vie professionnelle. Ces études débouchaient, en partie, sur des postes importants à la cour des princes phanariotes qui exigeaient la connaissance du français.

\subsection{Les précepteurs}

16 Force est de constater que la langue française gravitait autour du pouvoir de haut niveau. L'accès à son apprentissage était restreint à cette époque-là tout d'abord aux familles des princes et ce sont les secrétaires français qui jouaient souvent le rôle de précepteurs (Lebel 1955: 188-198) pour leurs enfants: Jean-Louis Carra auprès de Grégoire Ghika III, Clémaron dans la famille de Constantin Morousi, La Roche et Linchou auprès d'Alexandre Ypsilanti.

17 Par ailleurs, parmi ces secrétaires, émigrés ou voyageurs dans les principautés danubiennes, il y avait aussi des envoyés de l'Ambassade de France à Constantinople. À leur tour, les boïars ou nobles (en roumain, boier) font apprendre la langue française à leurs enfants pour que leur éducation ressemble à celle qu'on donnait aux jeunes princes. Le précepteur français qu'ils entretenaient dans leurs maisons était souvent chargé d'apprendre aussi à lire aux enfants des petits boïars (en roumain, boierinasi) ou à des jeunes paysans qui gravitaient autour de la résidence du maître pour accomplir diverses fonctions. Le français se propageait ainsi par le biais de l'éducation en famille, à l'aide des instituteurs français, émigrés de plus en plus nombreux vers la fin du XVIII siècle (pédagogues doués ou moins doués), mais également grâce à des Allemands, des Suisses, des Grecs qui se pressaient pour enseigner une langue qui, entre autres, rapportait une bonne rémunération.

18 Sur le territoire situé entre les fleuves Prut et Dniestr, le territoire qui englobe celui de la future République de Moldavie, l'enseignement du français suivait les mêmes tendances que dans toute la Moldavie. Dans les écoles privées, plus nombreuses que les écoles publiques, les élèves (le clergé, les boïars) apprenaient la langue française ou en langue française, au détriment du grec et du latin de l'ancienne méthode. Beaucoup de jeunes recevaient une éducation en famille avec des professeurs étrangers : des Grecs, des Allemands, des Français, etc. À cette époque où le français avait le statut de «langue internationale de l'Europe entière " (Iorga, 1918: 84), les boïars moldaves embauchaient les précepteurs français ou francophones pour donner une chance à leurs enfants de faire partie de l'élite administrative. Dans les capitales des principautés roumaines, Bucarest et Iassi, les princes «n'admettaient guère dans des postes un peu élevés que des personnages possédant cette langue » (Brunot 1967 : 941). 


\subsection{Le relais de la presse} langue pour leurs produits ou créations (littéraires, scientifiques ou culturelles, en général). Force est de constater que le français sert également les intérêts politiques des intellectuels roumains qui créent des contacts dans le cadre de leurs études à Paris, se rendent visibles et obtiennent l'appui de la France lors des événements politiques majeurs pour les Pays roumains comme ceux de 1848 et 1859.

La presse occidentale en langue française a largement contribué à la diffusion de cette langue en Moldavie. Implicitement, certes, car l'intérêt des abonnés était en premier lieu de s'informer sur l'actualité politique en Europe, ainsi que sur celle qui les concernait de plus près, et ensuite de faire la lecture des rubriques littéraires. En effet, dans la capitale moldave, autour de l'année 1780, les abonnés à la presse occidentale étaient relativement nombreux. À part le prince régnant, des cercles restreints de boïars, de prêtres, de médecins lisaient les titres français comme Le Mercure de France, Le Journal de Francfort et Le Journal littéraire.

En 1790 apparaît le premier journal bilingue roumain-français à Iassi, le Courrier de Moldavie, à l'initiative de Potiomkin (commandant des troupes russes installées à Iassi pendant la guerre russo-turque de 1787-1792), publication soutenue financièrement par les boïars moldaves. Le journal autrichien Wiener Zeitung mentionnait dans un de ses numéros que le Courrier de Moldavie était une rareté et qu'il avait deux colonnes, une dans la langue du pays et l'autre en français.

\subsection{Le contact avec les officiers}

Lors des conflits russo-turcs sur le territoire des principautés danubiennes, surtout en Moldavie, le contact avec des officiers russes ou des recrutés polonais, anglais, allemands et français dans l'armée russe - qui «parlaient tous plus volontiers le français que le russe »-a favorisé, selon Brunot, la diffusion de la langue française dans les salons roumains « où la conversation n'eut plus lieu que dans cette langue, surtout à partir de 1769 » (Brunot 1967 : 8). Les Russes, semble-t-il, « aimaient séjourner dans un pays où les accueillaient à bras ouverts des populations hospitalières » (ibid.) de Moldavie. En dehors du champ de bataille, les nobles russes «s'amusèrent de leur mieux » en apprenant à toute une jeunesse séduite par leur exemple à "aussi bien danser que prononcer le français " (Eliade $1898: 184$ ).

D'autres officiers, français ceux-ci, qui avaient dû quitter la Pologne après le premier démembrement de 1772 , se sont établis dans les principautés danubiennes, « aussi le français y fut-il de plus en plus appri et parlé [...]» (Brunot $1967: 8$ ).

3 Une continuité peut être observée dans la diffusion du français au XIX ${ }^{e}$ siècle où il garde encore son statut de langue élitaire, tout en devenant l'apanage de la couche sociale émergeante de l'intelligentsia.

Les intellectuels, issus des familles nobles, pour partie, la parlent ou l'emploient comme En Bessarabie ${ }^{5}$, les nombreux changements politiques - en commençant par l'année 1812 lorsqu'elle devient région ou oblasti (1812-1871) et ensuite goubernie (1871-1917) de l'Empire russe - font que la classe intellectuelle est assez hétérogène ${ }^{6}$. De ce fait, les influences sont multiples en ce qui concerne la présence et la diffusion de la langue française; elles s'exercent par l'intermédiation russe et roumaine, par la présence 
française qui s'intensifie suite à la politique du tsar qui facilite l'installation en Bessarabie d'une centaine de Français cherchant asile en Russie.

$\mathrm{Du}$ côté de l'enseignement du français en Bessarabie, sous la subordination départementale d'Odessa à l'époque (la «capitale » de la " Nouvelle Russie», selon le souhait de Catherine II), il est dispensé à Chisinau et dans d'autres villes plus importantes des différents districts, au niveau secondaire laïc ou religieux (écoles, collèges, gymnases, pensions, séminaires). La langue française figure parmi les matières obligatoires, ainsi que parmi celles facultatives (l'allemand et le russe étaient également étudiés, le plus grand nombre d'heures étant accordé au russe). Les émigrés de différentes origines installés en Bessarabie, dont les «Français au service de la Russie » (Salon 1981 : 96-97), y enseignaient le français. Plus tard, cet enseignement est dispensé par les autochtones.

Ceux qui souhaitent continuer à l'apprendre ou le parfaire partent à Odessa, à Iassi (deux villes avec une présence française croissante au XIX ${ }^{e}$ siècle), à Bucarest ou à Paris même, car les voyages et les études en France se pratiquent de plus en plus fréquemment.

Au XXe siècle, pour la période 1918-1940, lorsque la Moldavie fait partie de la Grande Roumanie, les données factuelles recueillies montrent que le développement de l'enseignement roumain, privé et public, a été propice à la diffusion du français. À l'instar du lycée de garçons «B. P. Hasdeu » de Chisinau, nationalisé par décret royal en 1918, les écoles moldaves continuent la tradition d'enseigner le français (à côté du latin et de l'allemand), malgré les nombreux changements de fonctionnement liés au régime politique. Ciobanu (1941: 94) note l'existence de deux lycées français à Chisinau, « tous deux presque exclusivement fréquentés par les israélites». L'école en français est perçue comme un "terrain neutre " à Chisinau où la situation sociolinguistique relève $\mathrm{du}$ conflit entre les Russes (ou les russophones) et les Roumains (ou les roumanophones) dans la Bessarabie de l'entre-deux-guerres. Le Service des œuvres françaises à l'étranger, à travers la mission universitaire et la section du livre français, intervient en Bessarabie pour une «rapide diffusion du français dans les régions nouvellement acquises à la Roumanie » (Godin 1998 : 10).

L'étude de la période 1940-1991, lorsque la Moldavie fait partie de l'URSS jusqu'à l'obtention de son indépendance le 27 août 1991, permet de constater que le français occupe une place importante parmi les autres langues (comme l'allemand, l'anglais, l'espagnol et l'italien). L'analyse des documents d'archives ${ }^{7}$ sur la création des premières facultés de langues étrangères dans les années 1940 fait ressortir que l'Institut pédagogique "A. Russo » de Balti était la principale institution moldave à enjeux importants dans la formation de professeurs de français en Moldavie et en URSS et, par conséquent, dans la diffusion ultérieure du français dans les écoles moldaves. En 1954, sa chaire de français compte onze enseignants, formés à Moscou, SaintPétersbourg, Kiev et Odessa. Cette institution d'enseignement supérieur aurait été le plus grand centre de l'URSS où l'on formait les professeurs de français, avec plus de 250 étudiants. Les premières promotions de diplômés ont fait émerger par la suite des professeurs de renom, auteurs de manuels, dictionnaires, grammaires et pionniers dans l'enseignement de plusieurs domaines disciplinaires de la linguistique française au sein des départements de français du milieu universitaire et de la recherche de Moldavie (Gutu 2006 : 37-40). 
lles étaient les raisons de la continuité de la diffusion prédominante du français en Moldavie à l'époque où Moscou favorisait plutôt l'enseignement de l'allemand et de l'anglais ? Une réponse possible serait l'héritage historique et une volonté politique de maintien du français dans le seul pays soviétique de langue latine, dans le cadre d'une politique conjoncturelle où Moscou s'engage devant la France à augmenter le nombre d'élèves qui apprennent le français. Les données statistiques indiquent pour l'année 1981 que $72 \%$ des élèves moldaves apprennent le français, pourcentage inchangé jusqu'en 1991.

31 Comment évolue la place du français de 1991 à aujourd'hui ? Existe-t-il une politique linguistique à l'égard du français en Moldavie?

\section{Existe-t-il une/des politique(s) linguistique(s) du français ?}

Le constat exprimé par différents chercheurs que la langue est " une affaire politique » et «donc une affaire d'État» (Klinkenberg 2000: 105), oriente ce travail vers la recherche d'indicateurs de l'action de l'État moldave et de ses institutions à l'égard de la langue française, avant et surtout après 1991, tout en tenant compte de la pluralité d'acteurs qui puissent y contribuer à différents niveaux. Cette problématique peu explorée par d'autres études a nécessité une recherche sur le terrain, auprès des acteurs politiques, éducatifs et sociaux susceptibles de nous offrir des éléments de réflexion. Un choix de méthodes complémentaires a été opéré pour recueillir des productions discursives diverses et des données factuelles à l'égard du français en Moldavie et essayer de comprendre le plus amplement possible la situation du français. Il s'agit notamment du recueil de différents types de textes comme les notes informatives, les arrêtés ministériels, les rapports d'activité, les programmes scolaires, ainsi que de productions discursives des acteurs institutionnels et sociaux. Grâce à cette diversité de sources et à l'articulation qui a pu être faite entre elles, une chronologie relative a pu être mise en place et a permis de voir comment se positionne l'État par rapport au français.

D'abord, la période avant 1991, où le français garde une position dominante grâce à la «tradition d'enseignement » qui est maintenue par les autorités éducatives moldaves. Ensuite, l'année 1991, où la Moldavie devient indépendante, amène un tournant dans l'enseignement des langues étrangères dû à la politique internationale d'ouverture sur la scène internationale. Dans ce contexte, plusieurs changements sont prévus dans la politique éducative, mais il n'y a aucune indication dans les textes officiels sur des objectifs ou des mesures qui concerneraient le français précisément, contrairement à l'anglais pour lequel les autorités commencent à pencher dans leurs discours. Cette politique générale d'absence de soutien à l'égard du français mais de soutien actif à l'anglais détermine dans cette deuxième période, de 1991 à 2011, le recul quantitatif progressif pour le français et un progrès stable pour l'anglais. Enfin, l'inversement des statistiques en faveur de l'anglais se produit en 2011. Cette situation où l'enseignement du français est fragilisé en Moldavie et où l'anglais le devance, dans un contexte de mondialisation économique, peut ne pas surprendre. Pourtant, une des spécificités de la Moldavie par rapport aux autres États voisins et/ou européens, c'est le maintien du français jusqu'en 2011 en première position dans le classement général.

Ces trois périodes établies, celles avant 1991, après 1991 et après 2011, sont valables pour la situation générale du français dans l'enseignement moldave. Le croisement des

Documents pour l'histoire du français langue étrangère ou seconde, 54 | 2015 
données statistiques et des discours recueillis montre qu'il existe des particularités géographiques et des différences en fonction de la langue d'enseignement que ce soit le roumain, le russe ou celle des minorités nationales, qui favorisent ou non le français. Ces particularités apparaissent comme un reliquat d'une situation ancienne et non pas comme un choix volontariste actuel de la part de l'État.

\section{Les acteurs de la francophonie en Moldavie}

Si l'on examine la langue française en Moldavie, il n'est guère possible de ne pas s'intéresser aux acteurs exogènes de la francophonie, présents dans cet espace après 1991. Leurs actions forment une question assez vaste et très peu thématisée et traitée. Il s'agit avant tout de la France à travers son ambassade et de l'Alliance française de Moldavie qui est un contributeur important pour le rayonnement du français, tant dans les faits que dans la perception des Moldaves. Ensuite, de l'OIF, organisation à laquelle la Moldavie a adhéré quelques années après son indépendance, qui trouve dans ce pays un terrain d'action propice pour soutenir le français à travers trois de ses partenaires : AUF, TV5Monde et AIMF. D'autres réseaux ou organisations où la Moldavie est représentée sont évoqués et discutés.

L'examen de nombreuses sources comme les rapports d'activités des acteurs, les documents de politique concernant le français, les traités ou accords de coopération, les articles de presse, les questionnaires réalisés en 2009, a permis de retracer leur action qui s'avère importante pour le français en Moldavie. Les autorités moldaves y sont associées. Pourtant, cela ne semble pas empêcher le recul du français et, surtout, ne remplace pas ce qui pourrait être une politique linguistique de l'État à l'égard de cette langue, langue qui présente des enjeux de développement pour l'État et les citoyens moldaves. Un développement qui pourrait se réaliser au travers des rôles importants du français particulièrement en tant que langue de la recherche, de la mobilité (académique et professionnelle), du développement économique et du rapprochement avec l'UE, ainsi que vecteur de la diversité linguistique et culturelle.

Enfin, si durant les siècles passés, la diversité d'acteurs contribuait à la diffusion du français, suivant une vague européenne due aux statuts et au prestige de cette langue, aujourd'hui ces acteurs se placeraient plutôt à contre-courant d'une "modernité » globalisante ou d'une mondialisation (libérale).

\section{BIBLIOGRAPHIE}

BRUNOT, Ferdinand (1967, $2^{\mathrm{e}}$ éd.). Histoire de la langue française, des origines à 1900 ou à nos jours. Paris : Librairie Armand Colin. T. 8 : Le français hors de France au XVIII ${ }^{\mathrm{e}}$ siècle, vol. I. (1934) et vol. II (1935).

CAMARIANO-CIORAN, Ariadna (1974). Les Académies princières de Bucarest et de Jassy et leurs professeurs. Institut d'Études Balkaniques de Thessalonique. 
CIOBANU, Stefan (1941). La Bessarabie : sa population, son passé, sa culture. Bucarest : Collection Études et recherches de l'Académie Roumaine, Imprimerie nationale.

COSTE, Daniel (1998). « 1940 à nos jours : Consolidations et ajustements ». Le français dans le monde, Recherches et applications : « Histoire de la diffusion et de l'enseignement du français dans le monde ", nº spécial, 75-95.

ELIADE, Pompiliu (1898). De l'influence française sur l'esprit public en Roumanie. Les origines. Étude sur l'état de la société roumaine à l'époque des règnes phanariotes. Thèse présentée à la Faculté des Lettres de Paris. Paris : Édition Ernest Leroux.

GHERVAS, Stella (2008). Réinventer la tradition. Alexandre Stourdza et l'Europe de la Sainte-Alliance. Paris : Éditions Honoré Champion.

GODIN, André (1998). Une passion roumaine. Histoire de l'Institut français de Hautes Études en Roumanie (1924-1948). Paris : L'Harmattan.

GUTU, Ana (2009). « La création littéraire francophone en République de Moldova ». Actes du colloque international « La Francopolyphonie : langue et culture françaises en Europe du SudEst », 24-35. En ligne : <https://app.box.com/shared/55x0v4pcxf> (05 février 2014).

GUTU, Ion (2006). « Aspects historiques de la francophonie moldave ». Actes du colloque international « La Francopolyphonie comme vecteur de la communication », 36-40. En ligne : <https://app.box.com/shared/5ifa4e01l3> (05 février 2014).

IORGA, Nicolae (1918). Histoire des relations entre la France et les Roumains. Paris : Payot.

KLINKENBERG, Jean-Marie (2000). «Les politiques linguistiques : pour qui ? pour quoi ? ». In Jacqueline Dematry-Warzee \& Jean Rousseau (dir.). Les Cahiers du CIEP : Français de l'avenir et avenir du français. Paris : Didier, 99-114.

LEBEL, Germaine (1955). La France et les Principautés danubiennes (du XVI ${ }^{\mathrm{e}}$ siècle à la chute de Napoléon I ${ }^{\mathrm{er}}$ ). Paris : Édition Presses Universitaires de France.

LEMNY, Stefan (2009). Les Cantemir : l'aventure européenne d'une famille princière au XVIII ${ }^{e}$ siècle. Paris : Éditions Complexe.

MOREL, Pierre (2008). Pour une Francophonie réaliste. Chisinau : Université Libre Internationale de Moldavie.

PARMENTIER, Florent (2010). Moldavie. Les atouts de la francophonie. Paris : Éditions Non Lieu. REY, Alain, DUVAL, Frédérique, SIOUFFI, Gilles (2011). Mille ans de langue française, histoire d'une passion. 2 vol. Paris : Éditions Perrin.

SALON, Albert (1981). L'Action culturelle de la France dans le monde: analyse critique. Thèse de doctorat, en 3 vol. Université Paris I Panthéon-Sorbonne.

TRUCHOT, Claude (2000). « 'L'espace externe' du français en Europe ». In Jacqueline DematryWarzee \& Jean Rousseau (dir.). Les Cahiers du CIEP : Français de l'avenir et avenir du français. Paris : Didier, 71-81.

TURCAN, Olga (2009). « La promotion de la francophonie en Moldavie par la coopération décentralisée (franco-moldave) ». Actes du Colloque international « Francopolyphonie : Langue et culture françaises en Europe du Sud-Est ». Chisinau : Université Libre Internationale de Moldova, 151-160. En ligne : <https://app.box.com/shared/3ntgbbj79l> (20-21 mars 2009).

TURCAN, Olga (2013). « Aspects des politiques linguistiques à l'égard du français en Moldavie : quelle(s) dynamique(s) ? ». Actes du $2^{\text {e }}$ Congrès européen de la FIPF « Vers l'éducation plurilingue en Europe avec le français. De la diversité à la synergie ». Prague. Vol. 1. Axe 1 : Politiques 
linguistiques, 163-174. En ligne : <http://prague2011.fipf.org/sites/prague2011.fipf.org/files/ actes_-prague_1._net.pdf> (08-10 septembre 2011).

\section{NOTES}

1. Dans ce travail, le nom de «Moldavie» renvoie au territoire de l'actuelle République de Moldavie (ou Moldova), pays frontalier de la Roumanie et de l'Ukraine.

2. Les Phanariotes sont des Grecs, en règle générale des lettrés, qui vivent dans le quartier de Constantinople appelé Phanar(i). Pour eux, la connaissance des langues est d'une importance majeure et, en premier lieu, la connaissance du français, qui est devenu après 1714 langue de la diplomatie. Sachant se rendre indispensables à la Porte, les Phanariotes obtiennent à partir de la deuxième décennie du XVIIIe siècle les postes les plus convoités - ceux de princes de la Moldavie (1711) et de la Valachie (1716) et cela jusqu'en 1821.

3. Nicolae Iorga (1871-1940) est un esprit encyclopédique qui a laissé une vaste œuvre. Professeur à l'université de Bucarest, il a enseigné également à la Sorbonne et au Collège de France. Il a été membre de l'Académie Roumaine et membre correspondant de l'Institut de France.

4. Durant les périodes comprises entre 1774-1782 et 1796-1797, Alexandre Ypsilanti a été prince de Valachie et de 1786 à 1788 , prince de Moldavie.

5. Bessarabie, une autre dénomination du territoire de la République de Moldavie, à l'exception de quelques régions frontalières du sud et du nord qui font à présent partie de la Roumanie et de l'Ukraine. Le nom Bessarabie provenant, semble-t-il, de la dynastie Basarab, qui avait dirigé les régions de Valachie et non pas de Moldavie, serait peu approprié, d'après Ghervas (2008:60).

6. À partir de nos recherches, nous tentons une sorte de description de la classe intellectuelle de Bessarabie d'après 1812 à partir de catégories que nous proposons : les autochtones, les Russes ou les envoyés des Russes pour mettre en place la politique impériale ; les autochtones « exilés » ou « émigrés 》 (dans les Principautés roumaines ou en Russie qui, à certaines époques, peuvent être considérées comme leur deuxième pays ou patrie), les « voyageurs » ou les « semi-nomades » qui changent souvent de ville (Chisinau - Odessa - Iassi, etc.) et y habitent, travaillent ou font des études/recherches pendant une certaine période ; les immigrés (y compris français) qui dans des contextes différents arrivent et s'installent en Bessarabie.

7. Il s'agit des documents des archives du ministère de l'Éducation de la République de Moldavie, consultés dans le cadre du travail documentaire effectué lors d'un déplacement à Chisinau, en novembre 2010.

\section{AUTEUR}

\section{OLGA TURCAN}

Université de Strasbourg 